\title{
Development of a high-rate tolerant HV-switching multi-wire proportional chamber and its readout electronics for DeeMe experiment
}

\author{
N. Teshima $*^{a}$, M. Aoki ${ }^{b}$, S. Mihara ${ }^{c}$, D. Nagao ${ }^{b}$, Y. Nakatsugawa ${ }^{c}$, H. Natori ${ }^{c}$, \\ H. Nishiguchi ${ }^{c}$, Y. Seiya ${ }^{a}$, Y. Takezaki ${ }^{a}$, N. D. Thong ${ }^{b}$, N. M. Truong ${ }^{b}$, K. Yamamoto ${ }^{a}$, \\ on behalf of the DeeMe Collaboration \\ ${ }^{a}$ Osaka City University \\ ${ }^{b}$ Osaka University \\ ${ }^{c} \mathrm{KEK}$ \\ 3-3-138 Sugimoto, Sumiyoshi-ku, Osaka-shi, Osaka, Japan \\ E-mail: teshimalocupc1.hep.osaka-cu.ac.jp
}

\begin{abstract}
The DeeMe experiment at Materials and Life Science Experimental Facility (MLF) of J-PARC is planned to search for muon-electron conversion processes with a single event sensitivity of one or two orders of magnitude better than the current upper limits. The Charged Lepton Flavor Violation (CLFV) such as the muon-electron conversion process is forbidden in the Standard Model (SM) and experimental observations of the CLFV provide clear evidence of new physics beyond the SM.
\end{abstract}

In the DeeMe experiment, the charged-particle tracking detectors, multi-wire proportional chambers (MWPCs), are irradiated in high-rate prompt-charged particles $\left(\cong 2 \times 10^{8}\right.$ particles / pulse) before signal electrons arrive at the MWPCs. With a standard design of the MWPC, the detector will be totally saturated and blinded for a while due to the huge amount of ions produced by the avalanche with such a high rate. In order to avoid the problem, we developed a novel technique based on the micro-cell structure of MWPC with HV switching of the potential wires to dynamically control the avalanche gas gain. The readout electronics is optimized to tolerate large prompt current and to cancel $1 / \mathrm{t}$ tail of the response to prompt-charged particles. In this paper, the details of the MWPC system will be presented.

Flavor Physics \& CP Violation 2015

May 25-29, 2015

Nagoya, Japan

*Speaker 
Development of a high-rate tolerant $H V$-switching multi-wire proportional chamber and its readout electronics for DeeMe experiment

\section{Introduction. The concept of DeeMe experiment}

Charged Lepton Flavor Violation (CLFV) processes are essentially forbidden in the Standard Model (SM) and have not been observed yet. But they occur naturally in many new physics beyond the SM. Experimental observations of the CLFV provide clear evidence of new physics.

DeeMe experiment is planned to search for the muon-electron conversion process in the nuclear field, $\mu^{-}+(A, Z) \rightarrow e^{-}+(A, Z)$, which is one of the CLFVs. The experiment will be conducted at J-PARC Materials and Life Science Experimental Facility (MLF).

The pulsed proton beam from $3 \mathrm{GeV}$ Rapid Cycling Synchrotron (RCS) is injected into the muon production target. Muons and the target atoms produce muonic atoms. The signal electrons of muon-electron conversion in the muonic atoms have monochromatic momentum $105 \mathrm{MeV} / c$. The electrons with momenta around $105 \mathrm{MeV} / \mathrm{c}$ are transported through the MLF MUSE H-line (under construction). After the transportation, the momenta of them are measured by the spectrometer magnet and four MWPCs located in front of and behind the magnet.

The DeeMe experiment will start with a single event sensitivity (S. E. S.) $\cong 1 \times 10^{-13}$ (for $1 \mathrm{MW}$ proton beam and $2 \times 10^{7}$ seconds) using a graphite target. It will be changed to a silicon carbide target with a S. E. S. $\cong 2 \times 10^{-14}$. The current upper limits of muon-electron conversion are $7 \times 10^{-13}$ for a gold target by the SINDRUM-II experiment at PSI and $4.6 \times$ $10^{-12}$ for a titanium target by the experiment at TRIUMF.

\section{The DeeMe detector}

\subsection{Requirements}

The MWPCs are irradiated in high-rate prompt-charged particles $\cong 2 \times 10^{8} /$ pulse with the pulse width of $200 \mathrm{~ns}$ (the maximum instant hit rate is $\sim 70 \mathrm{GHz} / \mathrm{mm}^{2}$ ) produced by the pulsed proton beam before the $105 \mathrm{MeV} / \mathrm{c}$ signal electrons arrive at the MWPCs. At such a high rate, a standard design of the MWPC will produce a huge amount of avalanche ions and be totally saturated, then blinded for a while. The DeeMe MWPCs are required to have:

1) high rate tolerance,

2) an ability to detect a signal electron soon after the prompt burst pulses of charged particles.

\subsection{Construction}

The final design of the MWPC is shown in Figure 1. There are 90 channels of $3 \mathrm{~mm}$ wide cathode strips in the $\mathrm{X}$ direction and 16 channels of $15 \mathrm{~mm}$ wide cathode strips in the $\mathrm{Y}$ direction. The active region is $250 \times 200 \mathrm{~mm}^{2}$. Anode wires and potential wires are tightened up alternately. The distance between an anode wire and a potential wire is $0.7 \mathrm{~mm}$. The gap between the anode plane and the cathode plane is $3 \mathrm{~mm}$.

To achieve the requirements mentioned in section 2.1, we develop a technique of highvoltage (HV) switching on potential wires of the MWPC. While the prompt-charged particles hit the MWPC, high voltage on the potential wires is swiched from $0 \mathrm{~V}$ (ground) to the voltage as 


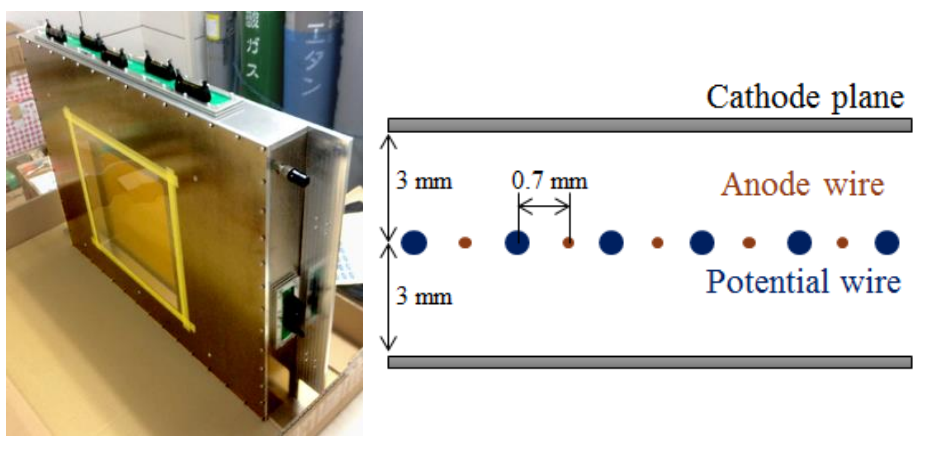

Figure 1: A photograph and a cross-section view of the final design MWPC.

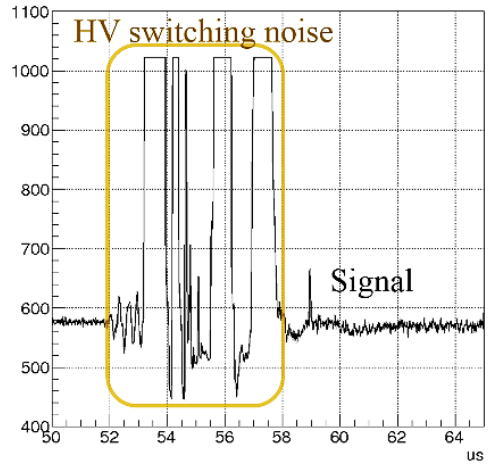

Figure 2: A waveform obtained in the test beam experiment.

high as that on the anode wires. Then potential gradient of the electric field in the MWPC is smaller and the avalanche gas gain is strongly suppressed during the switching period.

\subsection{Test beam experiment of the prototype}

Figure 2 shows a waveform obtained in the test beam experiment at Kyoto University Research Reactor Institute (KURRI) in January, 2015. A pulsed electron beam with intensity of the DeeMe experiment condition hits the prototype MWPC during the HV switching period. From the waveform, we see that the prompt burst pulse of charged particles is suppressed by the HV switching technique and that an electron signal by field emission in the accelerator is detected after the HV switching noise.

\section{Readout amplifier}

\subsection{Requirements and trial production}

The gain of the amplifier has to be enough to amplify small charge from the MWPC. At the same time, it needs large current tolerance for the signal of prompt-charged particles. The prompt burst pulse signal from the MWPC has long tail inversely proportional to the time. To avoid the amplifier saturation by the long tail, a pole-zero cancellation circuit is inserted in the amplifier. The readout circuits for the DeeMe experiment are required to have:

1) enough gain to amplify and large current tolerance for the signal of the prompt-charged particles,

2) large current tolerance for the charge induced by the HV switching technique on the potential wires of the MWPC,

3) an ability to cancel the long 1/t tail of the MWPC when the prompt burst pulse hits the MWPC.

We designed a readout circuit satisfying the requirements, which is shown in Figure 3, and manufactured a prototype of the amplifier. The amplifier circuit is called Radeka two-stage type and the original circuit design of this was taken from the system developed for the VENUS 


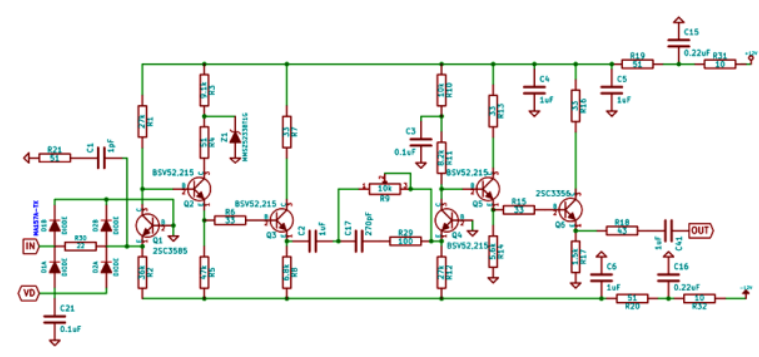

Figure 3: A circuit diagram of the Radeka two-stage-type amplifier for the DeeMe experiment.

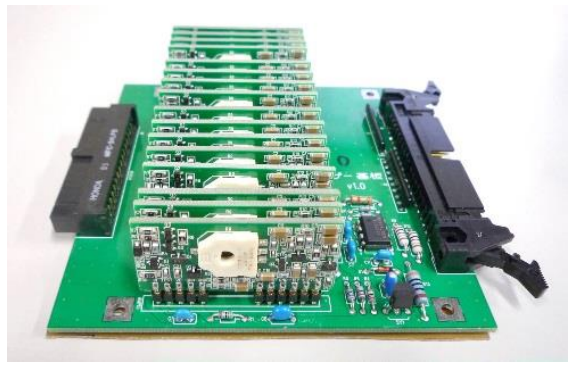

Figure 4: A photograph of 16 channels of the amplifier.

vertex chamber [3]. The Radeka type amplifier's stage consists of a common base and two common collectors and the Radeka two-stage type amplifier has two stages. A rectifier diode circuit in input of the amplifier protects the back circuits from the large current. A pole-zero cancellation circuit for the long $1 / \mathrm{t}$ tail is between the first stage and the second stage. The amplifiers work with $12 \mathrm{~V}$ and the power consumption of the 16 channel amplifiers is about $1 \mathrm{~W}$ when no charge inputs to them.

\subsection{Test of the prototype and mass production}

The test beam experiment at KURRI in January, 2015 (Section 2.3) confirms that a prototype of the amplifier has large current tolerance and an ability to cancel the long tail of the signal by pulsed electron beam with intensity of the DeeMe experimental condition. After the test beam experiment, we mass-produced 384 channels of the amplifier. Figure 4 shows the massproduced amplifier of 16 channels. The 16 channels of amplifier is integrated on the mother board. The mother board size is $100 \times 100 \mathrm{~mm}^{2}$.

\section{Summary}

The DeeMe experiment at J-PARC MLF is planned to search for muon-electron conversion processes in the nuclear field. In the experiment, the detector needs high rate tolerance and to be able to detect a signal electron for muon-electron conversion soon after the prompt burst pulse of charged particles producted by the pulsed proton beam. We are developing the MWPCs and its readout electronics satisfying those requirements. We will get ready to begin our experiment soon after MLF MUSE H-line construction in 2015-2016.

\section{References}

[1] M. Aoki, AIP Conference Proceedings 1441, 599 (2012).

[2] H. Natori, Nucl. Phys. B (Proc. Suppl) 284-250 (2014) 52-57.

[3] M. Morii, T. Taniguchi and M. Ikeno, "Development of a readout electronics system for the VENUS vertex chamber”, KEK Internal, 87-14 (1988). 